\title{
Clinical Preparedness in a Dental School Setting: A Collaborative Approach to Patient Care through Teaching, Learning, and Clinical Preparedness
}

\author{
Theano Eliopoulos*, Samantha Sauers and Sarah Pagni \\ School of Dental Medicine, Tufts University, Kneeland Street, Boston, MA, USA
}

*Corresponding author: Theano Eliopoulos, Assistant Professor, Practice Coordinator, School of Dental Medicine, Tufts University, Comprehensive Care, 1 Kneeland Street, Boston, MA, 02111, USA, Tel: 617-636-6894; E-mail: theano.eliopoulos@tufts.edu

Received: 08 Dec, 2020 | Accepted: 21 Dec, 2020 | Published: 28 Dec, 2020

Citation: Eliopoulos T, Sauers S, Pagni S (2020) Clinical Preparedness in a Dental School Setting: A Collaborative Approach to Patient Care through Teaching, Learning, and Clinical Preparedness. Int J Dent Oral Health 7(1): dx.doi.org/10.16966/2378-7090.348

Copyright: (C) 2020 Eliopoulos T, et al. This is an open-access article distributed under the terms of the Creative Commons Attribution License, which permits unrestricted use, distribution, and reproduction in any medium, provided the original author and source are credited.

\begin{abstract}
Background: At Tufts University School of Dental Medicine (TUSDM), the conclusion of second year didactic courses initiates the move to a more clinically driven course load and the simultaneous transition into providing patient care. This study investigated the attitudes and perceptions of dental students towards the Introduction to the Dental Patient (IDP) II and III courses, as well as clinical preparedness, as students transition from preclinical didactic courses to the clinic.
\end{abstract}

Methods: A survey of 14 questions was distributed in December 2019 to D21 students through Qualtrics, asking students' opinions of IDP courses and their confidence level with clinic. Data collection was anonymous. Statistics were calculated, along with Fisher's Exact Test, Spearman Correlation and Mann-Whitney U test.

Results: 50 responses were received. Twenty-three (46\%) of respondents reported feeling confident in their abilities as a dental practitioner in clinic at six months, and 17 (34\%) felt prepared at the 3-month mark. Students felt more instruction was needed regarding treatment planning, consultations, the handling of medically complex patients, for improvement in their clinical integration. Data did not find gender bias or correlation between previous clinical experience and student confidence in preparedness at the TUSDM clinic setting. There was a statistically significant positive correlation between when students felt prepared to treat patients in the clinic and their confidence in their abilities.

Conclusion: Survey responses show student attitudes and perceptions towards the curriculum and their confidence in a clinical setting in dental school. We will analyze these responses in order to help strengthen the curriculum to improve the clinical confidence and perceptions of the students prior to entering the clinical setting at TUSDM.

\section{Background}

The Commission of Dental Accreditation (CODA) defines as the goal of a dental education program "to develop graduates who have the capacity for life-long and self-directed learning and are capable of providing evidence-based care to meet the needs of their patients and of society." As defined on its standard 2-24, "at a minimum, graduates must be competent in providing oral health care within the scope of general dentistry" with the intent that "graduates should possess the basic knowledge, skills, and values to practice dentistry, independently, at the time of graduation." To this end, dental schools' curriculum should mirror the experiences of a general dentist in a general practice setting. The desired outcome is to graduate students who have confidence in the myriad of procedures they can expect to conduct as general dentists [1,2].

At most dental schools, the conclusion of second year didactic courses initiates the move to a more clinically driven course load and the simultaneous transition into providing patient care [3]. This is a significant yet stressful change for dental students. Shifting from the preclinical lab and rubber manikin heads to patient treatment is exciting, but it is an area of apprehension for dental students [4]. Dentistry concerns matters of human life, and patients hold dental professionals to a high standard in the delivery of quality care and professionalism under any and all circumstances thus, dentistry is a demanding career. The apprehension that overwhelms dental students prior to immersion into the clinical setting can result in a decreased self-esteem, which can lead to problems in professional life and potentially compromised patient care [5].

Effective teaching is crucial for clinical learning and retention for professional students [6]. This is especially true for dental students. It is imperative that students are provided with a positive clinical learning experience under guided instruction, to sculpt and enhance student opinions and perceptions on overall patient health. Clinical 
faculty should be trained in advising students on clinical techniques and procedures, uniting the aspects of teaching and learning, and have the ability to initiate appropriate changes in communication techniques, technical skills, and the attitudes and behaviors in daily practice in order to benefit patients [7].

A lecture course in which classroom learning evolves into the clinical application is crucial to a comprehensive dental education [8]. Dental students, who enter clinic more prepared with an understanding of the systems, practices, and procedures, will most likely perform better upon transitioning into the clinical setting [3]. The importance of including student input in education is accepted as a key component of processes used to monitor the quality of academic programs [9]. This is especially ideal in the dental school clinical setting where curriculum and practices are constantly evolving [1].

Prior to entering the clinic setting, students at Tufts University School of Dental Medicine (TUSDM) take a course called Introduction to the Dental Patient (IDP). At the start of second year, they are enrolled in IDP II, which begins the transition from aspects of dentistry taught in basic science didactic courses and the preclinical laboratory, into clinical practice. Through lectures and workshops, this course builds upon the skills acquired previously, for the successful transition into a knowledgeable student dentist. They are taught the concepts of Infection Control, Periodontology, Radiology and the Electronic Health Record (Axium), paired with HIPAA training. Prior to entering the clinic in June, these students are enrolled in IDP III. Here students are taught the concepts of Oral Diagnosis, Caries Detection, Ethics and Professionalism, Cultural Awareness and specialty specific workshops, to prepare them to treat patients when they are in the dental school clinic. The goal of the IDP courses is to guide students into beginning patient care at TUSDM, to ensure their success when they graduate and enter the real world.

The aim of this study was to test the attitudes and perceptions of dental students regarding the efficacy of the Introduction to the Dental Patient II and III courses (IDP), as well as their clinical preparedness in transitioning from preclinical didactic courses to patient care in the clinic. It was hypothesized that dental students feel that they lack confidence and are not as prepared as they should be when they enter clinical practice at the end of their second year, and that they would prefer more guidance and detailed instruction to correct this.

\section{Subjects, Materials and Methods}

This study was approved by the Tufts Health Sciences Institutional Review Board, study \#1911031. The authors conducted a content validity of the survey by having Tufts Dental clinical faculty review the questions for usefulness and appropriateness, and face validity by having Tufts senior dental students review them for clarity, simplicity, usefulness, and appropriateness. The questionnaire was distributed online, using Qualtrics, to195 consenting TUSDM third year dental students treating patients in the months of November and December 2019.There students would have been in the clinic for approximately 6 months.

Our questionnaire consisted of fourteen questions using the fivepoint Likert scale (Strongly Agree, Agree, Neutral, Disagree, Strongly Disagree) except when noted otherwise. The questions asked students if the IDP II and III courses were helpful with integration to the clinic, and if the lectures were similar to how students approach procedures in clinic. They asked if the lectures were detailed and informative for doing exams, operative dentistry, periodontics, prosthodontics. Also, did students want more lectures on clinic specific protocols, such as the
Axium electronic record, treatment planning, phasing of procedures, or completing consultations in oral pathology or medicine? Would a clinical syllabus or a clinic review course be helpful to assist with preparedness and confidence upon entering clinic? Additionally, students were asked if the Objective Structured Clinical Examination (OSCE) administered prior to their entrance into clinic was thorough and representative of protocols and procedures in the clinic, and if students felt confident in their abilities as dental practitioners in the clinic, at this point in their clinical career. Students were asked at what point upon entering clinic they felt prepared to treat patients, immediately, within 2 weeks, one month, three months or six months, along with student demographics. After emailing the questionnaire to 195 third-year dental students at TUSDM, we allowed six weeks for responses, with one reminder email going out two weeks before the deadline.

Statistics were used to evaluate the data obtained from the 14-question survey, using Stata 13.1 (StataCorp LP, College Station, TX). After evaluating the responses, we further analyzed the results using Fisher's Exact Test, Spearman Correlation, and Mann-Whitney $\mathrm{U}$ test, to compare the questions and responses.

\section{Questionnaire}

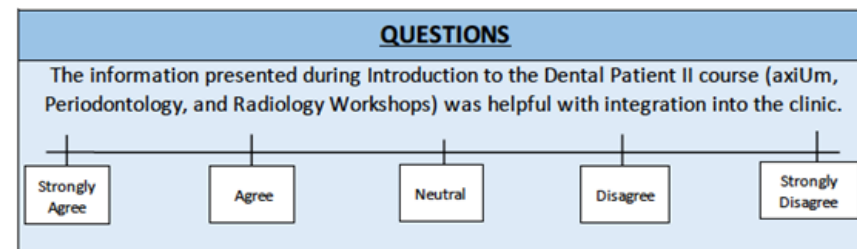

The information presented during Introduction to the Dental Patient III course (Specialty based lectures, Radiology Workshops, etc.) was helpful with integration into the clinic.

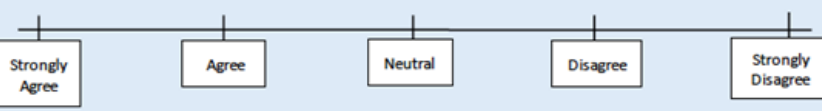
The lectures taught during Introduction to the Dental Patient II and III courses were similar \begin{tabular}{|c|c|c|c|}
\hline $\begin{array}{c}\text { Strongly } \\
\text { Agree }\end{array}$ \\
\hline Aeree
\end{tabular}

The lectures taught during Introduction to the Dental Patient II and III courses were detailed and informative for the applicable discipline. Exams (D0150, D0120, New Patient Encounter)

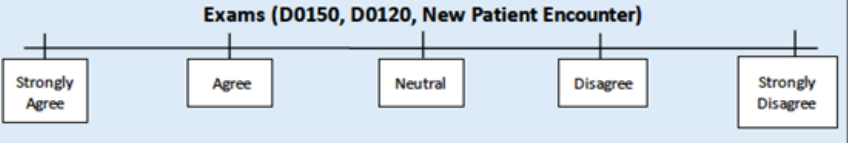

The lectures taught during Introduction to the Dental Patient II and III courses were detailed and informative for the applicable discipline.

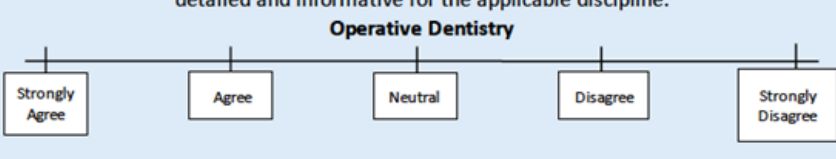

The lectures taught during Introduction to the Dental Patient II and III courses were detailed and informative for the applicable discipline.

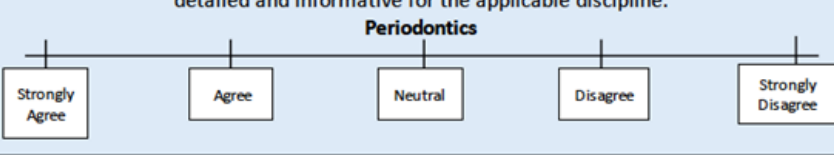

The lectures taught during Introduction to the Dental Patient II and III courses were detailed and informative for the applicable discipline.

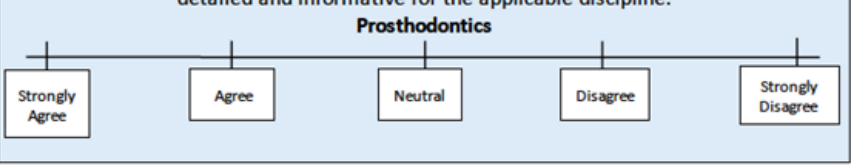



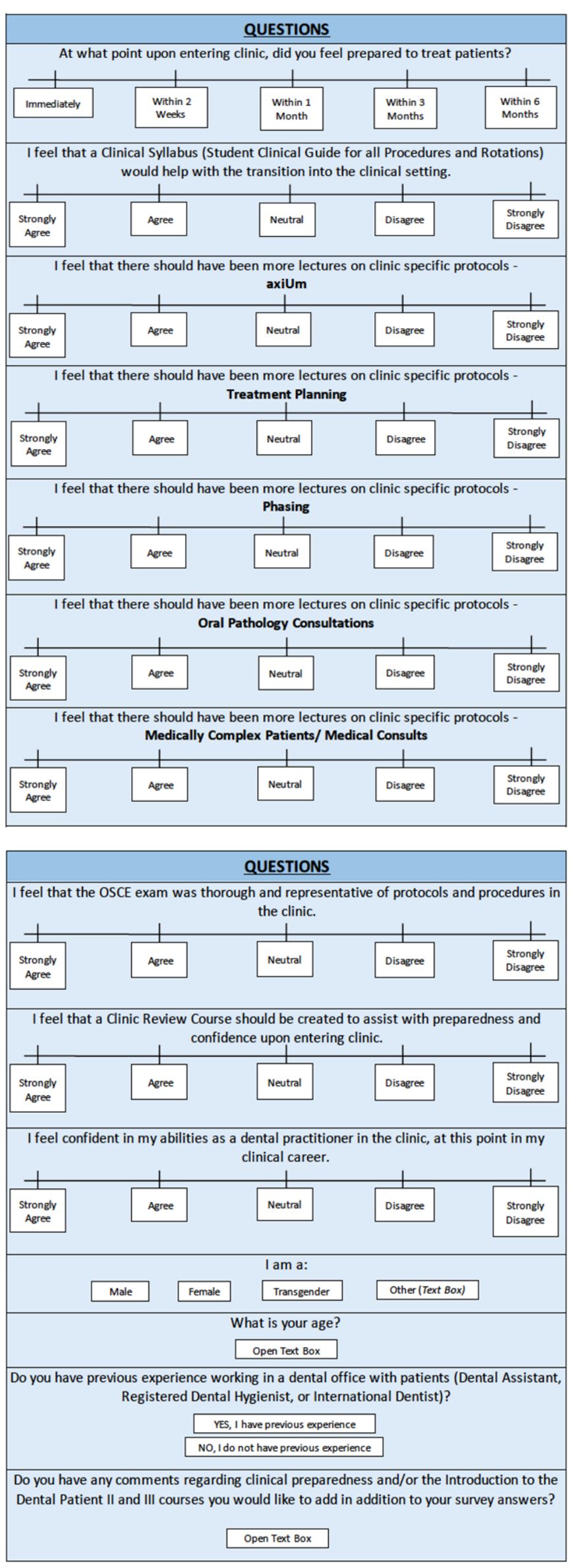

\section{Results}

50 responses were received. Respondents ranged in age from 23 to 38 years old (average 26.9 years old) and 18 out of the $50(36 \%)$ had previous experience working in a dental office. There was no difference in age among students with previous dental experience and those without.

After approximately 6 months of clinical experience, $46 \%$ of the 50 respondents stated that they felt confident in their clinical abilities. There were 11 students (22\%) who did not feel confident after 6 months in the clinic (Table 1). Interestingly, previous experience was not correlated with students' confidence in their clinical abilities ( $P$ Value $=0.22$ ).

Very few respondents (10\%) reported that they felt prepared to treat patients in the first two weeks on the clinic floor. The highest percentage of respondents $(34 \%)$ stated that they felt prepared to treat patients within 3 months; with the remaining respondents (28\%) indicating that it took up to six months before they felt prepared (Figure 1).

There was no statistically significant association between gender or age and when students felt prepared to treat patients in the clinic. However, their level of confidence in their abilities as a dental practitioner was positively associated (Spearman's rho $=0.5254$ ) with when students felt prepared to treat patients.

Concerning student age and the time frame where they felt confident to see patients in the clinic, with Spearman's rho $=-0.2288$, we discovered there was a weak negative correlation. This implies that older students felt slightly less confident in seeing patients in the clinical setting over time.

Overall, when asked if the information presented in the IDP II course, taught earlier in the second academic year, was helpful with their integration into the clinical setting, 36\% of respondents agreed. $64 \%$ of them agreed that the information from the IDP III course, taught closer to the start of clinic, was helpful with the clinical integration (Figure 2). Twenty-two respondents (44\%) stated that the lectures were not similar to how they approach procedures in the clinic, while 13 respondents (26\%) stated that they were similar.

When asked about the detailed lectures and if the material was informative and detailed, a majority in each of the sections stated that they agreed with the statement. Respondents felt lectures on Operative Dentistry and Periodontics were helpful, but less so with Oral Exams and especially with Prosthodontics. When asked if students desired more lectures on certain aspects of the clinic, respondents preferred more information on the electronic patient record (Axium) (88\%), treatment planning (96\%), and phasing of treatment (80\%). A trend appears to exist when evaluating results for these clinic specific protocols and procedures (Figure 3).

An OSCE is given to dental students at the end of their second year, after the IDP II and III lectures and prior to entering the clinic. This

Table 1: Respondent is confident in their abilities as a dental practitioner at the time of the questionnaire-November/December 2019.

\begin{tabular}{|l|c|c|}
\hline & Percentage & \# Respondents \\
\hline Strongly Agree & $4 \%$ & 2 \\
\hline Agree & $42 \%$ & 21 \\
\hline Neutral & $32 \%$ & 16 \\
\hline Disagree & $18 \%$ & 9 \\
\hline Strongly Disagree & $4 \%$ & 2 \\
\hline
\end{tabular}




\section{When Did You Feel Prepared to Treat Patients?}

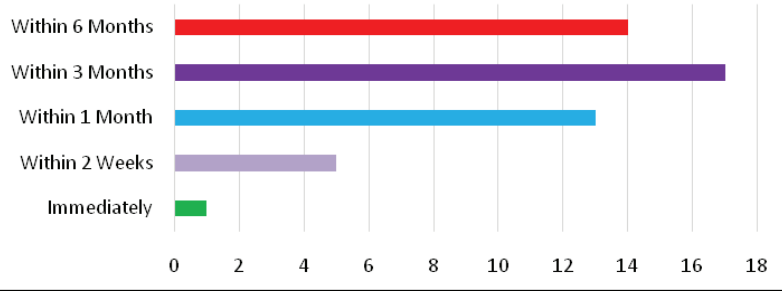

Figure 1: Respondents were asked about when they felt prepared to treat patients after they entered the clinical setting. Most felt prepared after 3 months while very few felt prepared in the first two weeks.

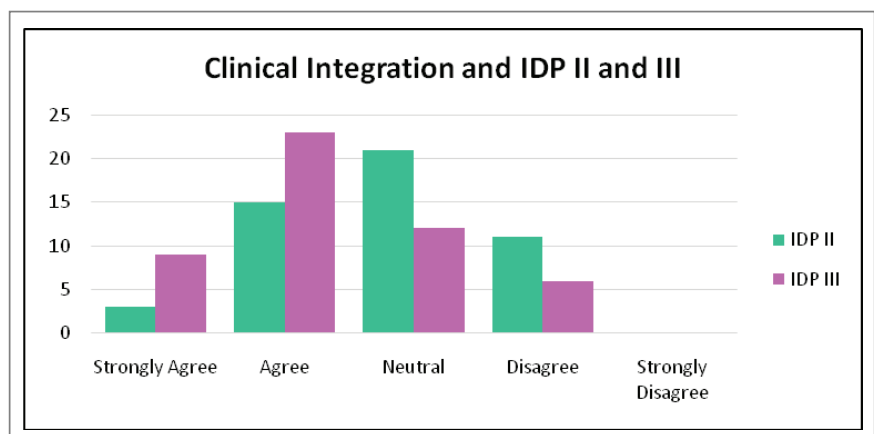

Figure 2: Respondents were asked if the lectures and information they were presented in the Introduction to the Dental Patient II and III courses were helpful with their clinical integration. Most respondents agreed that it was helpful.

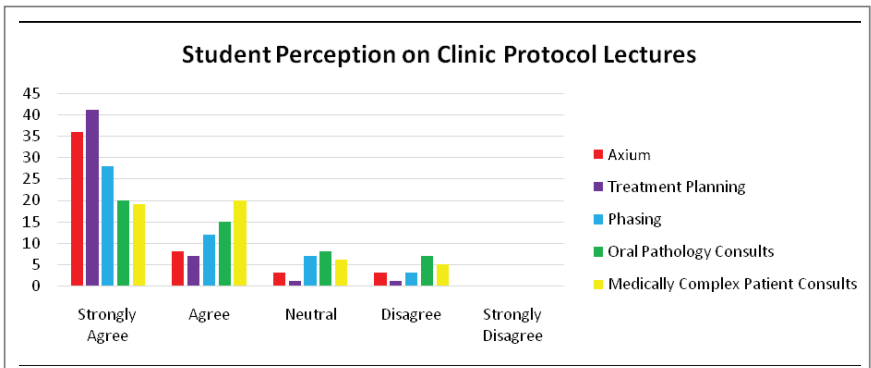

Figure 3: Respondent perception on the helpfulness of clinic specific lectures and protocols offered in Introduction to the Dental Patient II and III courses, and the extent of the information given. Topics listed are those that give students the hardest time upon entering clinic.

is a situational exam where students apply their didactic knowledge to the simulated clinical presentations placed before them. The majority $(71.43 \%)$ of respondents felt that this OSCE was not representative of the procedures and protocols in the clinic. Sixteen students $(32.65 \%)$ strongly disagreed, and 19 (38.78 \%) students disagreed (Figure 4).We only received 49 responses for this question.

A clinical review course and syllabus has never been implemented within the TUSDM undergraduate curriculum to aid in clinical integration. Therefore, students were asked if a clinical syllabus should be created to help with clinical transition, and if a review course would

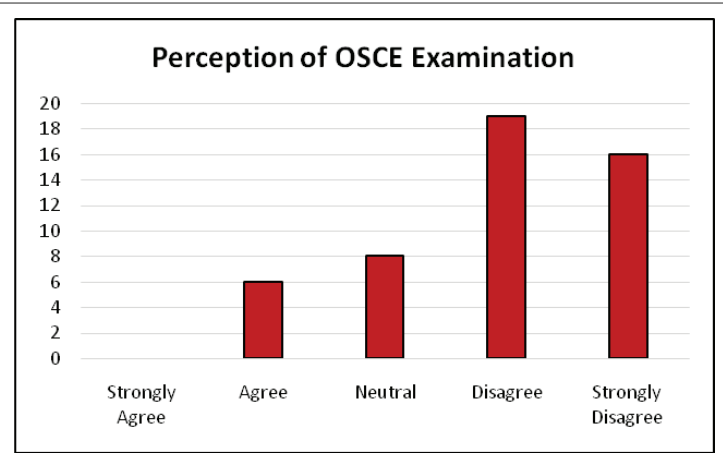

Figure 4: Respondent perception on OSCE. Students were asked if the OSCE that they took prior to entering the clinic is representative of protocols and procedures that are seen in the clinic.

help. Thirty-four respondents (68\%) strongly agreed on having a clinical syllabus to help them transition from manikin to patient care (Figure 5)

\section{Discussion and Conclusion}

This study assessed the attitudes and perceptions of third year dental students and their clinical confidence and preparedness. It was determined that, although students had the perception of preparedness and confidence as a clinical practitioner within six months of treating patients, more was needed to help increase that confidence and instill it earlier in their clinical experiences. In particular, certain subjects were more difficult to comprehend in the IDP II and III courses, especially the Axium electronic record and the clinic protocols to follow for clinical oral exams and the discipline of Prosthodontics.

The results show that age and gender do not play a factor in a dental student's confidence level, or even the time at which they feel confident treating patients upon entering the clinical setting as a new practitioner in their third year of dental school. Surprisingly, there was no significant difference in student confidence in their clinical abilities, between those with previous dental experience and those without it. We found that students who felt more prepared had more confidence in their abilities, and felt they were ready to treat patients at an earlier time frame than their classmates. This is an important finding regarding curriculum development because even though clinical confidence and competency do not necessarily correlate, increasing self confidence in "clinical activities are considered an essential element in teaching dentistry [10]".

Another study found that "complex procedures that were least practiced scored the lowest in overall mean (student) confidence" [11]. These findings can help TUSDM structure its curriculum to enable greater competence in dental skills and hopefully increase student confidence as well. In another study it was found that the students' "practical experiences were more important than the teaching method used to achieve students' perceived competence" [12].

At TUSDM OSCEs are being implemented earlier in the curriculum and at more frequent intervals, to better gauge student knowledge in real world scenarios. It is hoped that this method of testing will engage students in a more holistic approach to learning, which may translate into greater competence. Also, engaging students in the clinical realm at an earlier stage, for example having first year students actively input data in patient charts as they assist their classmates with dental exams, will benefit their clinical learning. 


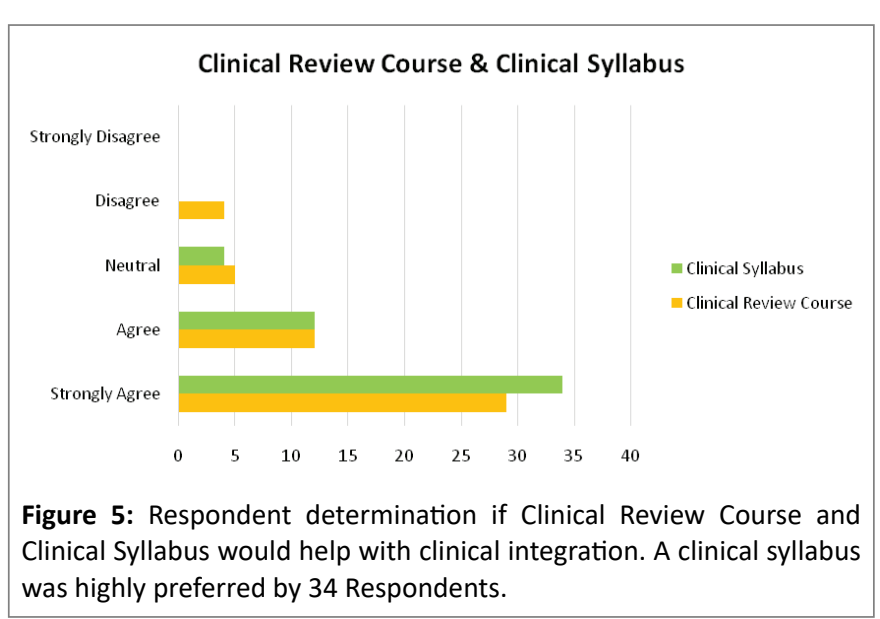

A limitation of this study is the inability to compare the student perception of their clinical preparedness with concrete metrics of their progress in clinic, to verify that these correlated well. It is possible that students who felt confident and clinically prepared lacked technical skills, compared to those who were more tentative. Also, our study's focus was on TUSDM's IDP courses, which are not identical to courses offered at other dental schools to prepare students to enter clinic.

In conclusion, the authors identified that more needed to be done to increase the clinical preparedness and the confidence of students entering the clinical realm of their training at TUSDM. Future studies will be conducted to assess the impact of a clinical syllabus and a review course on students' clinical confidence. The authors suggest that more clinically relevant tests in the form of OSCEs be added to the preclinical curriculum.

\section{References}

1. Haden NK, Hendricson WD, Kassebaum DK, Ranney RR, Weinstein G, et al. (2010) Curriculum Change in Dental Education, 2003-09. J Dent Educ 74: 539-557.
2. Manakil J, George J (2012) Self-perceived Work Preparedness of the Graduating Dental Students. Eur J Dent Educ 17: 101-105.

3. Serrano CM, Botelho MG, Wesselink PR, Vervoorn JM (2018) Challenges in the Transition to Clinical Training in Dentistry. Eur J Dent Educ 22: e451-e457.

4. Haralur SB, Al-Malki AE (2014) Student Perception about Efficacy of Preclinical Fixed Prosthodontic Training to Facilitate Smooth Transition to Clinical Context. J Educ Health Promot 3: 73.

5. Shah SIA, Ahmed M (2013) Medical Students' Anxiety on Beginning Clinical Studies. Al Am een J Med Sci 6: 195-201.

6. Goldie J, Dowie A, Goldie A, Cotton P, Morrison J (2015) What Makes a Good Clinical Student and Teacher? An Exploratory Study. BMC Med Educ 15: 40

7. Schonwetter DJ, Lavigne, S, Mazurat R, Nazarko O (2006) Students' Perceptions of Effective Classroom and clinical Teaching in Dental and Dental Hygiene Education. J Dent Educ 70: 624-635.

8. Ferguson MB, Sobel M, Niederman R (2002) Preclinical Restorative Training. J Dent Educ 66: 1159-1162.

9. Henzi, D, Davis E, Jasineicius R, Hendricson W (2007) In the Students' Own Words: What are the Strengths and Weaknesses of the Dental School Curriculum? J Dent Educ 71: 632-645.

10. Thilakumara IP, Prathibhani KU, Rasnayaka SGK, Abeysundara SP, Jayasinghe RM (2020) Does the Level of Confidence Exhibited by Dental Students Predict the Outcome of Complete Denture Therapy? Int J Dent 2020: 9752925.

11. Gilmour ASM, Welply A, Cowpe JG, Bullock AD, Joes RJ (2016) The Undergraduate Preparation of Dentists: Confidence Levels of Final Year Dental Students at the School of Dentistry in Cardiff. Br Dent J 221: 349-354.

12. Montero J, Dib A, Guadilla Y, Flores J, Santos JA, et al. (2018) Dental Students' Perceived Clinical Competence in Prosthodontics: Comparison of Traditional and Problem-Based Learning Methodologies. J Dent Educ 82: 152-162. 